\title{
Association analysis of the ACTN3 R577X polymorphism and complex quantitative body composition and performance phenotypes in adolescent Greeks
}

\author{
Colin N Moran ${ }^{1}$, Nan Yang ${ }^{2}$, Mark ES Bailey ${ }^{1}$, Athanasios Tsiokanos ${ }^{3}$, Athanasios Jamurtas ${ }^{3}$, \\ Daniel G MacArthur ${ }^{2}$, Kathryn North ${ }^{2}$, Yannis P Pitsiladis* ${ }^{* 1}$ and Richard H Wilson ${ }^{1}$
}

\begin{abstract}
${ }^{1}$ Institute of Diet, Exercise and Lifestyle (IDEAL) and Division of Molecular Genetics, Faculty of Biomedical \& Life Sciences, University of Glasgow, Glasgow, UK; ${ }^{2}$ Institute for Neuromuscular Research, Children's Hospital at Westmead, Faculty of Medicine, University of Sydney, NSW, Australia; ${ }^{3}$ Department of Physical Education and Sport Science, University of Thessaly, Trikala, Greece
\end{abstract}

The functional allele (577R) of $A C T N 3$, which encodes human $\alpha$-actinin-3, has been reported to be associated with elite athletic status and with response to resistance training, while the nonfunctional allele (577X) has been proposed as a candidate metabolically thrifty allele. In a study of 992 adolescent Greeks, we show that there is a significant association $(P=0.003)$ between the ACTN3 R577X polymorphism and $40 \mathrm{~m}$ sprint time in males that accounts for $2.3 \%$ of phenotypic variance, with the $577 \mathrm{R}$ allele contributing to faster times in an additive manner. The R577X polymorphism is not associated with other power phenotypes related to $40 \mathrm{~m}$ sprint, nor with an endurance phenotype. Furthermore, the polymorphism is not associated with obesity-related phenotypes in our population, suggesting that the $577 \mathrm{X}$ allele is not a thrifty allele, and thus the persistence of this null allele must be explained in other terms.

European Journal of Human Genetics (2007) 15, 88-93. doi:10.1038/sj.ejhg.5201724; published online 11 October 2006

Keywords: obesity; sprinting; Caucasians; thrifty gene

\section{Introduction}

Two calcium-insensitive $\alpha$-actinins are expressed in muscle ( $\alpha$-actinin- 2 and $\alpha$-actinin-3; ACTN2, ACTN3). These form a lattice structure, anchoring actin-containing thin filaments at the sarcomeric Z-line. ${ }^{1} \alpha$-Actinin- 2 is found in skeletal and cardiac muscle, while $\alpha$-actinin- 3 is found only in skeletal muscle, and almost exclusively in the primarily anaerobic, glycolytic type $2 \mathrm{~b}$ fibres. ${ }^{2}$ In addition to their structural role, $\alpha$-actinin- 2 and $\alpha$-actinin- 3 also interact

*Correspondence: Dr YP Pitsiladis, Institute of Diet, Exercise and Lifestyle (IDEAL), Faculty of Biomedical \& Life Sciences, University of Glasgow, Glasgow G12 8QQ, Scotland, UK. Tel: +44 141330 3858; Fax: + 44141 330 2915; E-mail: Y.Pitsiladis@bio.gla.ac.uk

Received 2 May 2006; revised 24 July 2006; accepted 25 August 2006; published online 11 October 2006 with proteins involved in a variety of signalling ${ }^{3}$ and metabolic pathways. ${ }^{4,5}$

The ACTN3 codon 577 polymorphism (R577X; dbSNP rs1815739) originated as a result of a loss-of-function nonsense mutation replacing arginine codon 577 (577R) with a premature stop codon (577X). The nonsense allele produces no detectable $\alpha$-actinin- 3 protein. ${ }^{6}$ This allele is found in every human population tested, implying that it has an ancient origin within the human lineage and that both alleles may have been retained through balancing selection. In no other mammal, including the chimpanzee, has polymorphism for null alleles of ACTN3 been demonstrated. ${ }^{7}$ Few studies of the impact of ACTN3 genotype on human phenotypes have been carried out. Genetic variation in ACTN3 has been reported to be associated with elite athletic status ${ }^{8}$ and response to resistance training, ${ }^{9}$ and 
ACTN3 has been proposed as a candidate for a metabolically 'thrifty' gene. ${ }^{3}$ Among elite Caucasian athletes in Australia, XX homozygotes are under-represented in power athletes, including sprinters, relative to the general population, ${ }^{8}$ with no XX homozygotes being found among 32 sprint Olympians. This effect was seen in both male and female subjects. The XX genotype was present at a significantly higher frequency in female elite endurance athletes, suggesting that the $577 \mathrm{X}$ allele may be advantageous for endurance performance. However, no significant association was found in male endurance athletes. Similar findings have been reported for Finnish athletes. ${ }^{10}$

The differences in effect of the R577X polymorphism on phenotype in these different circumstances could be due to differences in muscle metabolism engendered by the presence or absence of $\alpha$-actinin-3. ${ }^{3}$ Under the hypothesis that the 577X allele has persisted as a metabolically 'thrifty' allele, it would be predicted that reduced levels or the absence of $\alpha$-actinin-3 from a subset of muscle fibres would result in more efficient energy storage or use of energy reserves. ${ }^{3,11}$ To test this hypothesis, and explore the functional relationships between R577X and physical- and performance-related phenotypes in an unselected population, we carried out an association analysis using 992 adolescent Greeks previously phenotyped ${ }^{12,13}$ for body composition-, strength/power- and endurance-related traits. Studying young individuals can in some circumstances reveal genotype-phenotype associations in complex traits more effectively than in adults because confounding effects of the environment have had less time to take effect. ${ }^{14}$

\section{Materials and methods}

The sample population has previously been described in detail. ${ }^{12,13}$ Healthy subjects were drawn from 10 urban and rural schools surrounding Trikala in central Greece. The study was approved by the Glasgow University Ethics Committee and local Greek authorities with written informed consent and parental approval obtained for all subjects. Overall compliance was over $90 \%$. Body composition phenotypes measured were BMI, triceps and subscapular skinfolds; power/strength-related phenotypes were handgrip strength, sitting basketball throw, vertical jump, $40 \mathrm{~m}$ sprint and agility run; as an endurance-related phenotype, the shuttle run test, a proxy for aerobic capacity $/ \mathrm{Vo}_{2}$ max, ${ }^{15-17}$ was employed. All these tests required little specialist equipment and so could be easily carried out in schools on a large scale. Detailed protocols for the basketball throw, $40 \mathrm{~m}$ sprint, agility run and shuttle run tests are given in the Supplementary Material (Section 1). All assessments were carried out by trained individuals following a period of protocol standardisation. At entry to the study, subjects completed a health questionnaire, and another documenting their participation in regular organised physical activity both inside and outside of school. Individuals with up to two hours of organised physical activity per week (the compulsory gym class) were classified as 'Inactive', those with $2-4 \mathrm{~h}$ per week as 'Mildly Active', those with $4-6 \mathrm{~h}$ per week as 'Intermediately Active' and those with more than $6 \mathrm{~h}$ per week as 'Highly Active'. This classification gave a roughly comparable number of male subjects in each physical activity level group (124 inactives, 157 mildly actives, 97 intermediately actives and 134 highly actives). For female subjects there were 205 inactives, 117 mildly actives, 76 intermediately actives and 54 highly actives. For ethical and practical reasons, we were unable to include an objective measure of puberty.

DNA extraction was carried out as described previously. ${ }^{13}$ Genotypes were determined using the TaqMan SNP Assay C_590093_1 (Applied Biosystems, Melbourne, Australia). The accuracy of the assay was validated in $\sim 10 \%$ of the samples using a previously published RFLP technique, ${ }^{7}$ a new RFLP assay (see Supplementary Material Section 2) or direct sequencing.

All statistical analyses were carried out using Minitab 13.32. We chose to account for the effects of age by grouping individuals according to single-year age classes, and for gender differences by analysing male and female subjects separately. To maintain equally large numbers among age groups, 11- and 12-year-olds were grouped together, and 17- and 18-year-olds were grouped together. Data for each gender and age group were tested separately for normality using the Ryan-Joiner test ${ }^{18}$ and subsequently transformed by logarithmic $\left(\log _{10}\right)$ transformation. The transformed data were then standardised for age by conversion to $z$-scores within year groups. All analyses were performed using $z$-scores; however, for presentation, $z$ scores were reverse-transformed to measurements corresponding to the 17- to 18-year-old group. Differences between genotype groups for investigated phenotypes were assessed using one-way ANOVA.

The critical value ( $\alpha$-value) of $P$, at which significance was accepted, was corrected for multiple testing (in the ANOVA tests) using the Dunn-Sidak ${ }^{19}$ method as implemented at the Simple Interactive Statistical Analysis ${ }^{20}$ website (SISA; http://home.clara.net/sisa/) and taking correlations between phenotypes into account (mean correlation 0.103; see Supplementary Material Section 3). The corrected $\alpha$-value used was $P<0.004$. Differences judged to be significant were further assessed to investigate the relationship between genotype and phenotype distribution using correlation analysis. Genotypes were assigned 'dummy variable' values according to the genetic model (additive allelic effects, R-allele dominant or X-allele dominant) being tested. Values assigned to each genotype in the correlation analysis for the Additive genetic model were 0 , 0.5 and 1 , representing homozygotes for one allele (RR), 
heterozygotes (RX) and homozygotes for the other allele $(\mathrm{XX})$, respectively; for the completely dominant genetic models, the corresponding values were $0,0,1$ or $0,1,1$, respectively, depending on which allele was being tested for dominance. The proportion of the genetic contribution to phenotypic variance explained by each genetic model was estimated by expressing $r^{2}$ from the correlation analyses (taken as an estimate of percentage variance explained under the model) as a percentage of the variance explained by genotype effects in the model-free ANOVAs. This proportion was compared for each model to predict the most accurate model tested.

To establish whether the genetic effects were influenced by the habitual physical activity level of the subjects, analyses were performed including the physical activity level groups (defined above) with the established genetic model in General Linear Model (GLM)-ANOVAs. GLM modelling allows both qualitative and quantitative predictor variables to be assessed as factors explaining quantitative dependent phenotypic distributions simultaneously. Similar tests were used to assess whether stage of pubertal development was a confounding factor in the analysis, by subdividing the male and female groups into 'younger' (age 11-13 years) and 'older' (age 14-18 years) subgroups after converting the data to height-corrected $z$-scores (rather than age-corrected $z$-scores).

The influence of genotype on the distribution of phenotypes in the study population was assessed by calculating odds ratios (ORs) and visualised using normal probability plots. For significant genotype-phenotype associations (as determined by ANOVA), male and female populations were divided into top, pooled-middle and bottom quartiles of the phenotype distribution. Odds ratios were calculated ${ }^{21}$ as the likelihood of individuals of a given genotype being in a selected quartile divided by the likelihood of their being in the remaining quartiles. Significance of the odds ratios was calculated using $2 \times 2 \chi^{2}$-tests and 95\% confidence intervals. Normal probability plots were produced using the default method in Minitab 13.32.

\section{Results}

In total, 1198 subjects were recruited from schools in central Greece, of whom 1084 were included in the analyses after collation of phenotypic data. Genotyping of buccal cell DNA was successful in 992 individuals (525 male, 467 female). R577X genotype distributions $(\mathrm{RR}=34 \%, \mathrm{RX}=48 \%, \mathrm{XX}=18 \%)$ were similar to those previously reported ${ }^{8}$ in a Caucasian Australian population and were consistent with Hardy-Weinberg equilibrium $\left(\chi_{\text {[df }=1]}^{2}=0.358 ; P=0.550\right)$; allele frequencies in the whole sample were $f_{(R)}=0.58$ and $f_{(X)}=0.42$.

Genotypic associations between the R577X polymorphism and measures of physical- and performance-related phenotypes were assessed by ANOVA using age-based $Z$-scores. Males and females were analysed as separate populations. Full analyses are presented in Table 1. Statistically significant $(P<0.004$; Sidak-corrected $\alpha$-value $)$ effects of genotype on $40 \mathrm{~m}$ sprint time were observed in male subjects but not female subjects (Table 1). Using post hoc $t$-tests, male subjects with both RR and RX genotypes had lower (faster) $40 \mathrm{~m}$ sprint times than individuals with the XX genotype (Table 1), but the difference between RR and RX means was not significant. We found no association between R577X genotype and other strength/powerrelated phenotypes (handgrip strength, basketball throw, vertical jump and agility run), nor did we see any association with the shuttle run results (as a proxy for aerobic capacity) suggesting that R577X does not influence endurance performance in this unselected adolescent population. We also observed no association with measures of body composition (BMI, triceps skinfold and subscapular skinfold), which would have indicated a possible 'thrifty' effect of the polymorphism on metabolism.

To investigate further the pattern of association of genotype with $40 \mathrm{~m}$ sprint time (shown in Figure 1) the data were tested against three genetic models (additive allelic effects, R-allele completely dominant and X-allele completely dominant) using correlation analysis. The Additive allelic model, in which the mean of the RX heterozygote group is modelled as being exactly midway between the means of the RR and XX homozygote groups, explained the largest portion of the genetic variance. An additive model is mechanistically the most attractive model, where increasing the dosage of a particular allele linearly alters the relative size of its effect. The Additive allelic model explains $96 \%$ of the genetic variance, the Rallele dominant model $79 \%$ and the X-allele dominant model 54\%. More complex genetic models of partial dominance would account for the remaining portions of the genetic variance.

Established associations (one-way ANOVA) were investigated further using GLM-ANOVA. No associations were established in female subjects; therefore GLM-ANOVA analyses were performed to test for interactions in male subjects only between the $40 \mathrm{~m}$ sprint time association data under the Additive allelic genetic model, physical activity level, school attended and age. No significant effect of physical activity level alone was found, nor was there any interaction effect between genotype and physical activity level (effect of R577X, $P=0.019$; of physical activity level, $P=0.205$; of their interaction, $P=0.265$ ). A predictable effect of age was noted (faster times recorded in older age groups), but no interaction between genotype and age (R577X, $P=0.018$; age, $P<0.001$; interaction, $P=0.344$; data normalised by height), suggesting that the influence of genotype is unaffected by pubertal stage. Which school each subject attended did influence $40 \mathrm{~m}$ sprint time, but there was no significant interaction $(P=0.466)$ between 
Table 1 ANOVA analysis of associations between R577X genotype and body composition-, strength/power- and endurancerelated phenotypes in male and female subjects

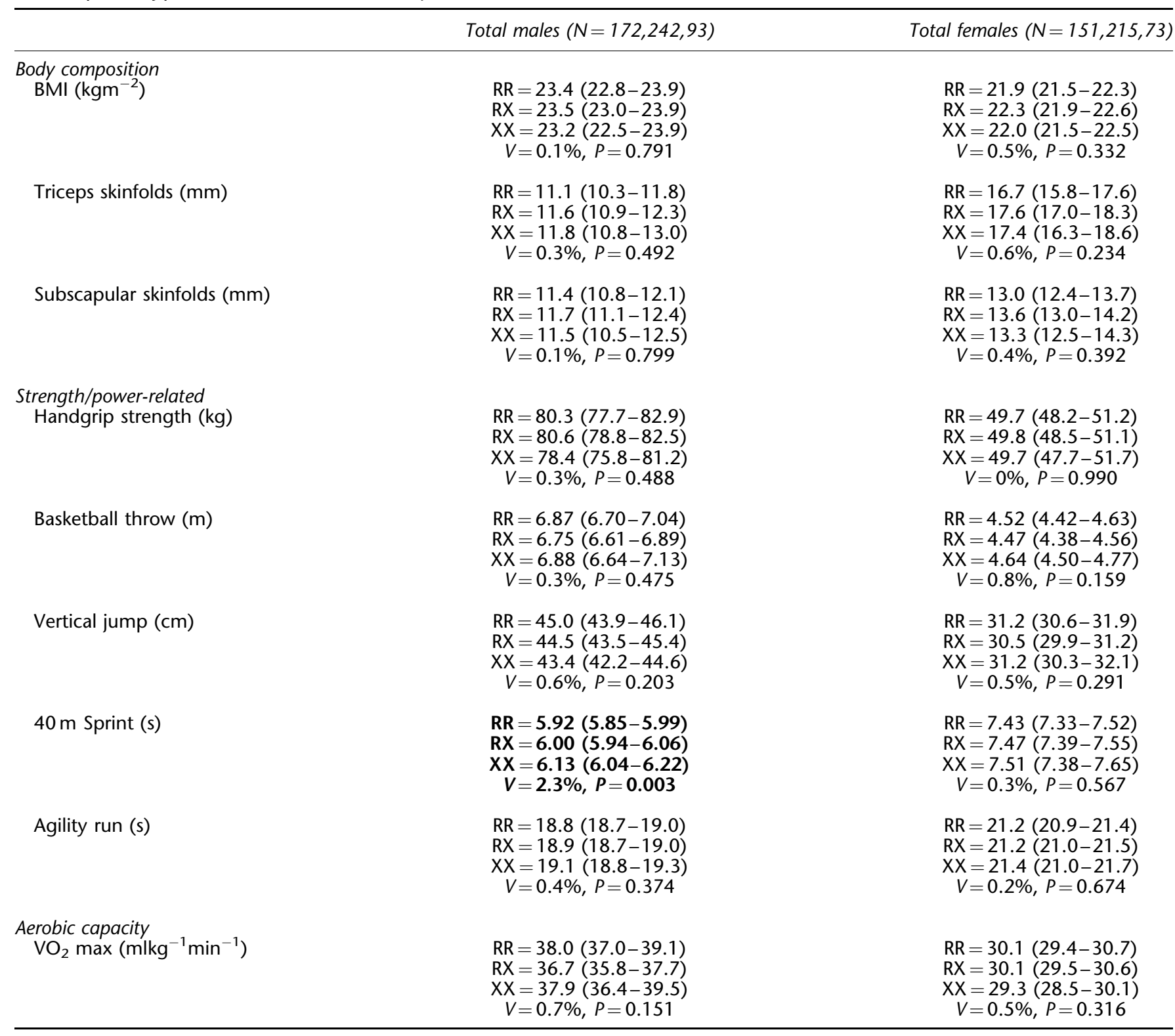

No. of individuals of each genotype is given in the column headings (for RR, RX and XX genotypes, respectively). For each test, means and their $95 \%$ confidence intervals are shown (to 3 significant figures) for each genotype as well as percentage variance explained in ANOVA ( $V$ ) and its associated probability $(P)$. The reported means and confidence intervals have been converted back from $z$-scores (used for age-correction) to the equivalent measurements in 17- to 18-year-olds. Tests significant after a Sidak correction $(P<0.004)$ are shown in bold. Note that smaller values for $40 \mathrm{~m}$ sprint and agility run are faster.

school and R577X genotype. When the effect of school was included in the model, the association with R577X genotype remained $(P=0.001, V=2.6 \%)$, showing that the effect of genotype was similar in all schools and that, therefore, school differences in measuring $40 \mathrm{~m}$ sprint time cannot explain the observed evidence for genotypephenotype association. No association between activity level and genotype was found $\left(\chi^{2}=2.97, \mathrm{df}=6, P=0.812\right)$, nor between school and genotype $\left(\chi^{2}=24.60, \mathrm{df}=18\right.$, $P=0.137)$.

The distribution of $40 \mathrm{~m}$ sprint times within each R577X genotype group is shown in Figure 2 where cumulative frequencies are plotted on a normal probability scale against $z$-scores. A normally distributed variable gives a straight-line relationship when plotted this way. This plot illustrates the differing means and phenotype distributions 


\begin{tabular}{|c|c|c|c|}
\hline Genotype & $\mathbf{N}$ & Mean & ANOVA \\
\hline $\mathrm{RR}$ & 172 & 5.92 & $\mathrm{P}=0.003$ \\
\hline $\mathrm{RX}$ & 242 & 6.00 & $\mathrm{~V}=2.3 \%$ \\
\hline $\mathrm{XX}$ & 93 & 6.13 & \\
\hline \multicolumn{4}{|c|}{ DIII ZIID } \\
\hline $\mathrm{RX}$ & \multirow[t]{2}{*}{ ZII } & $Z 7 Z$ & \\
\hline $\mathrm{XX}$ & & $D Z$ & DIIIID \\
\hline
\end{tabular}

Figure 1 Effect of R577X genotype on $40 \mathrm{~m}$ sprint times of total male subjects. Mean $40 \mathrm{~m}$ sprint times (Z-scores) back-transformed using the 17/18-year-old mean and standard deviation are plotted (black squares), together with $95 \%$ confidence limits of the mean (hatched bars), for each of the three genotypes, RR, RX and XX. ANOVA results (from Table 1 ) are also shown for ease of interpretation. $N=$ number within group, $\mathrm{P}=$ probability, $V=$ percentage observed variance explained by genotype.

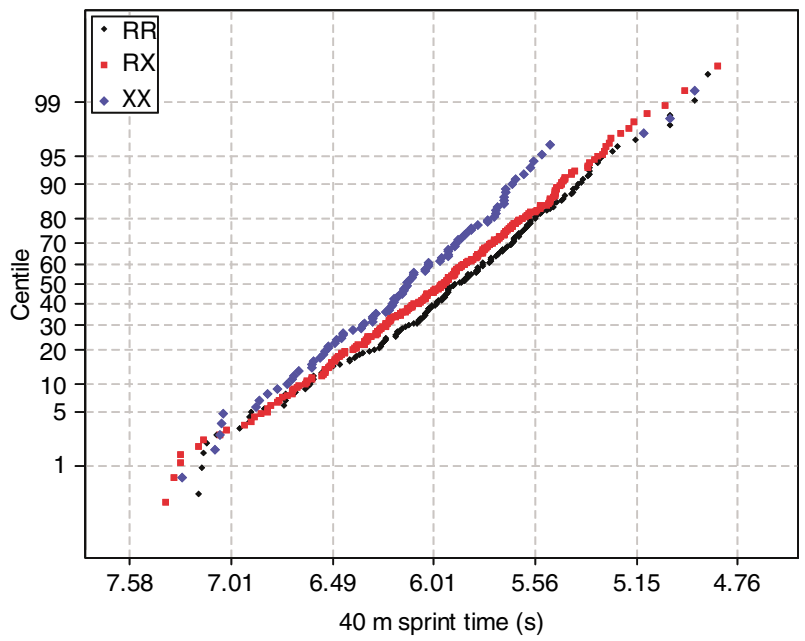

Figure 2 Normal probability plot for male $40 \mathrm{~m}$ sprint times by ACTN3 R577X genotype. Centiles were calculated for each genotype separately and plotted against $z$-score for each individual using Minitab 13.32. For illustrative purposes, the $z$-scores used for the scale have been back transformed to equivalent 17- to 18-year-old male times. Note that all $z$-scores were multiplied by -1 so that higher centile values represent quicker times, this also means that quicker times (on the $X$ axis) are further to the right. $N$-values are 172, 242 and 93 for RR, RX and XX, respectively.

for the three genotypes and shows that while performances for all three genotype groups approximate to normal distributions, at the fast end of the distribution for the $\mathrm{XX}$ genotype group, there are three outliers whose performance is approximately one standard deviation better than predicted from the remainder of the $\mathrm{XX}$ genotype distribution. Even allowing for these three XX outliers, the difference between genotype groups in the distributions becomes steadily more marked towards the faster end of the performance distribution.

Thus, the effects of genotype are seen most clearly at the faster end of the distribution and this is highlighted by the odds ratios (OR) for individuals of different genotypes falling into the low and high quartiles. RR homozygotes are significantly over-represented $(\mathrm{OR}=1.7 ; 95 \% \mathrm{CI} 1.1-2.5$; $P=0.013)$ and $\mathrm{XX}$ homozygotes under-represented $(\mathrm{OR}=0.3 ; 95 \% \mathrm{CI} 0.1-0.7 ; P=0.003)$ in the low (faster) quartile group for $40 \mathrm{~m}$ sprint time; in contrast, RR homozygotes are not significantly under-represented $(\mathrm{OR}=0.7 ; 95 \% \mathrm{CI} 0.4-1.1 ; P=0.090)$ nor are $\mathrm{XX}$ homozygotes significantly over-represented $(\mathrm{OR}=1.6 ; 95 \% \mathrm{CI}$ $0.9-3.0 ; P=0.098$ ) in the high (slower) quartile group. The differential effect size at either end of the distribution cannot be explained by an interaction between ACTN3 genotype and physical activity level on $40 \mathrm{~m}$ sprint time (GLM-ANOVA above).

\section{Discussion}

Our results demonstrate that genotype at the ACTN3 R577X polymorphism influences sprinting ability in an unselected population of Caucasian origin. The association of sprint performance in an unselected group of adolescent males confirms the associations found in elite sprinters of Caucasian origin, ${ }^{8}$ but the data do not reveal an association in a large sample of unselected adolescent female subjects. This is probably explained by differences between untrained adolescents and elite sprint athletes in terms of their exercise environments and muscle physiology, which may modify the interaction with genotype at the R577X polymorphism.

The distribution of SNPs around the R577X polymorphism, and the presence of 577X in all African and nonAfrican populations investigated so far, ${ }^{7}$ suggest that the polymorphism has existed for a considerable amount of evolutionary time and that balancing selection may have been involved in its maintenance. In this study we show a complexity of phenotype associated with the R577X polymorphism. We have confirmed an advantageous effect on sprinting speed for the 577R allele, but without any observable effect of this allele on other predominantly strength/power-related phenotypes. The polymorphism produces no observable effect on an aerobic capacity-based proxy for endurance. Similarly, our studies on elite East African endurance athletes failed to find any association with R577X (Yang N, MacArthur DG, Wolde B et al. ACTN3 genotype is not associated with elite endurance athlete status in Ethiopians and Kenyans. American College of Sports Medicine Annual Meeting, Nashville, USA. Med Sci Sport Exer, 2005; 37: S472, personal communication). Nor does the polymorphism produce an observable association with measures of body composition, phenotypes that might have demonstrated compensatory advantages for the 577X allele (eg metabolic 'thriftiness', expressing itself in the modern Western cultural environment as increased adiposity or BMI). Our sample size is sufficient to detect a quantitative trait association with a polymorphic locus if 
the locus explains $\geq 1.2 \%(P \leq 0.05)$ or more of the trait variance. The underlying properties of the R577X polymorphism that could produce this complexity of phenotype are unclear, especially as no other power/strength phenotype is affected. The sprinting phenotype is associated with power generation by repeated cycles of muscle contraction, in contrast to phenotypes associated with force generated by a single muscle contraction event (eg vertical jump). The molecular advantage of the 577R allele to phenotypes associated with cyclical generations of force (involving repeated contractions and relaxations) could be either structural (eg stabilisation of the Z-line in the myofibrils) or physiological (eg differential recruitment of nonstructural proteins to the Z-line). One possible explanation for the advantage of the 577R allele, that it confers protection against muscle damage during repetitive force generation, predicts as a consequence a structurally nonadaptive functional state for the $577 \mathrm{X}$ allele that could reasonably be predicted to be exposed to negative selection in a precivilisation environment. The compensatory advantage of the 577X allele that could therefore account for its persistence may lie in other aspects of muscle structure and function, but any connection with metabolic 'thriftiness' remains elusive.

We have shown that the ACTN3 R577X polymorphism is associated with a complex phenotypic characteristic in an unselected population, namely sprinting ability. This finding is consistent with previous studies that have reported association between the ACTN3 577R allele and elite sprint athlete status. In this nonelite population there was no evidence, however, for association with other power/strength-related phenotypes involving single muscle contraction events, suggesting that R577X is influencing the cyclical component of sprinting rather than strength or power generation. We also investigated the hypothesis that the $577 \mathrm{X}$ null allele might have been selected on the basis that it contributes to a metabolically 'thrifty' phenotype. We found no evidence that R577X genotype is associated with endurance- or obesity-related phenotypes. Further study should be directed at elucidating the molecular mechanisms by which variation in ACTN3 can affect muscle physiology.

\section{Acknowledgements}

This research was supported in part by a grant from the MRC/BBSRC associate programme in human nutrition research (\#17/D17566). We have no conflicts of interest to declare. The cooperation of all schools and subjects is greatly appreciated.

\section{References}

1 Squire JM: Architecture and function in the muscle sarcomere. Curr Opin Structural Biol 1997; 7: 247-257.

2 North KN, Beggs AH: Deficiency of a skeletal muscle isoform of alpha-actinin (alpha-actinin-3) in merosin-positive congenital muscular dystrophy. Neuromuscular Disord 1996; 6: 229-235.

3 MacArthur DG, North KN: A gene for speed? The evolution and function of alpha-actinin-3. Bioassays 2004; 26: 786-795.

4 Rakus D, Mamczur P, Gizak A, Dus D, Dzugaj A: Colocalization of muscle FBPase and muscle aldolase on both sides of the Z-line. Biochem Biophys Res Commun 2003; 311: 294-299.

5 Chowrashi P, Mittal B, Sanger JM, Sanger JW: Amorphin is phosphorylase; phosphorylase is an alpha-actinin-binding protein. Cell Motility Cytoskeleton 2002; 53: 125-135.

6 Vainzof M, Costa CS, Marie SK et al: Deficiency of alpha-actinin-3 (ACTN3) occurs in different forms of muscular dystrophy. Neuropediatrics 1997; 28: 223-228.

7 Mills MA, Yang N, Weinberger RP et al: Differential expression of the actin-binding proteins, alpha-actinin-2 and-3, in different species: implications for the evolution of functional redundancy. Human Mol Genet 2001; 10: 1335-1346.

8 Yang N, MacArthur DG, Gulbin JP et al: ACTN3 genotype is associated with human elite athletic performance. Am J Hum Genet 2003; 73: 627-631.

9 Clarkson PM, Devaney JM, Gordish-Dressman H et al: ACTN3 genotype is associated with increases in muscle strength in response to resistance training in women. J Appl Physiol 2005; 99: $154-163$.

10 Niemi AK, Majamaa K: Mitochondrial DNA and ACTN3 genotypes in Finnish elite endurance and sprint athletes. Eur J Hum Genet 2005; 13: 965-969.

11 Neel JV: Diabetes mellitus - a thrifty genotype rendered detrimental by progress. Am J Hum Genet 1962; 14: 353-362.

12 Moran CN, Vassilopoulos C, Tsiokanos A et al: The associations of $A C E$ polymorphisms with physical, physiological and skill parameters in adolescents. Eur J Hum Genet 2005; 14: 332-339.

13 Moran CN, Vassilopoulos C, Tsiokanos A et al: Effects of interaction between angiotensin I-converting enzyme polymorphisms and lifestyle on adiposity in adolescent Greeks. Obes Res 2005; 13: 1499-1504.

14 Maes HH, Beunen GP, Vlietinck RF et al: Inheritance of physical fitness in 10-yr-old twins and their parents. Med Sci Sport Exer 1996; 28: 1479-1491.

15 Leger L, Gadoury C: Validity of the 20-m shuttle run test with 1-min stages to predict $\mathrm{Vo}_{2}$ max in adults. Can J Sports Sci 1989; 14: $21-26$.

16 Leger LA, Lambert J: A maximal multistage 20-M shuttle run test to predict $\mathrm{VO}_{2}$ max. Eur J Appl Physiol Occup Physiol 1982; 49: 1-12.

17 Leger LA, Mercier D, Gadoury C, Lambert J: The multistage 20 metre shuttle run test for aerobic fitness. J Sports Sci 1988; 6: $93-$ 101.

18 Ryan Jr TA, Joiner BL: Normal Probability Plots and Tests for Normality. PA, USA: Statistics Department, The Pennsylvania State University, 1976.

19 Sokal RR, Rohlf FJ: Biometry: The Principles and Practice of Statistics in Biological Research. New York: WH Freeman and Company, 1995.

20 Uitenbroek DG: SISA Binomial. 1997 〈http://home.clara.net/sisa/ binomial.htm > Accessed 20051 April.

21 Armitage P, Berry G: Statistical Methods in Medical Research. Oxford: Blackwell Scientific Publications, 1994.

Supplementary Information accompanies the paper on European Journal of Human Genetics website (http://www.nature.com/ejhg) 